\title{
Bond Strength of Metal and Ceramic Brackets on Resin Nanoceramic Material With Different Surface Treatments
}

\author{
Mehmet Kara' (D), Özgür Demir² (D), Mehmet Doğru³ (D) \\ 'Department of Orthodontics, Inonu University School of Dentistry, Malatya, Turkey \\ 2 Private Practice, Istanbul, Turkey \\ ${ }^{3}$ Department of Orthodontics, Dicle University School of Dentistry, Diyarbakır, Turkey
}

Cite this article as: Kara M, Demir Ö, Doğru M. Bond Strength of Metal and Ceramic Brackets on Resin Nanoceramic Material With Different Surface

Treatments. Turk J Orthod 2020; 33(2): 115-22.

ABSTRACT

Objective: This study aimed to evaluate the effects of different surface conditoning methods on surface texture and shear bond strength (SBS) of brackets bonded to resin nanoceramic material.

Methods: Ceramic specimens were divided into two groups as metal brackets and ceramic brackets. In each group, the following five subgroups were conditoned with orthophosphoric acid (OPA), hydrofluoric acid (HFA), silica coating with Cojet, Nd: Yag laser, and Femtosecond ( $\mathrm{Fs}$ ) laser. Extra samples were used for scanning electron microscopy and 3D profilometer evaluation.

Results: All surface conditioning methods caused optimum or higher SBS. Metal brackets had higher SBS than porcelain brackets, but this difference reached statistical significance only in Fs laser group. OPA caused surface modification comparable to HFA because of polymer content of resin nanoceramic. Although Fs laser and Cojet conditioning caused optimum or higher SBS, surface damage of these methods to the resin nanoceramic specimens clearly seen on 3D profilometer.

Conclusion: HFA and Nd: Yag laser are effective surface conditioning methods for resin nanoceramics. OPA combined with silane application caused optimum SBS and can be used as an alternative to HFA. Surface texture changes should be considered to determine surface damage while deciding the optimum surface conditioning method for ceramics other than SBS.

Keywords: Dental materials, lasers, orthodontic brackets

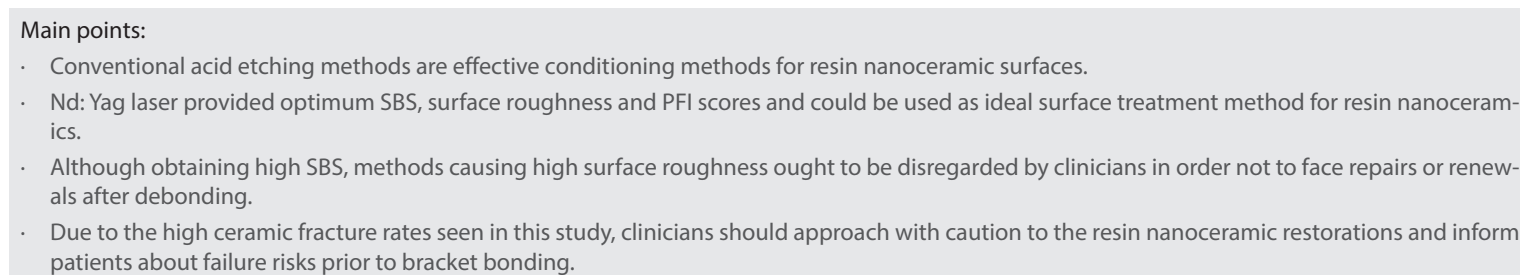

\section{INTRODUCTION}

With the current increase in adult patients seeking orthodontic treatments, orthodontists are more concerned about bonding orthodontic brackets other than enamel surfaces. Bonding ceramic surfaces are one of the reasons for this concern because a certain conditioning protocol could not be applied because of the material composition differences (1). Different mechanical and chemical conditioning methods and their combinations have been suggested to overcome this problem (2). Mechanical methods such as sandblasting and roughening with diamond burs require special tools and might cause damage to ceramic surfaces (3), chemical methods such as hydrofluoric acid (HFA) has found to be harmful and can cause tissue irritation (4). 
In recent years, lasers are used to overcome these disadvantages. Studies have been conducted using $\mathrm{CO}_{2}$, Er: Yag, $\mathrm{Nd}$ : Yag, and Femtosecond (Fs) lasers, and researchers stated that these have been useful alternatives for conventional conditioning methods $(5,6)$.

The demand for aesthetic restorations with the advances in software and milling devices has encouraged the research and development processes of more advanced porcelain systems using computer-aided design and computer-aided manufacturing (CAD/CAM) (7). Resin-matrix CAD/CAM ceramics have been developed to combine the advantageous properties of ceramics, such as durability and color stability, with those of composite resins, such as improved flexural properties and low abrasiveness (8). Composite resin nanoceramic CAD/CAM blocks consist of a polymeric matrix reinforced by nanohyrbrid ceramic fillers containing $71 \%$ by weight (7). Industrial fabrication of these materials at a high temperature and high pressure significantly improves their mechanical behavior $(9,10)$. To the best of our knowledge there, has been no report on bond strength of brackets bonded to resin nanoceramic CAD/CAM material.

There are many shear bond strength (SBS) studies in the literature, suggesting different conditioning methods for bonding brackets to ceramic surfaces using scanning electron microscopy (SEM) to determine surface texture changes $(2,5,11)$. These studies mostly focused on attaining the highest SBS results and disregarded the possible irreversible surface texture changes, which are secondary to the conditioning methods. 2D SEM images alone are insufficent to assess these changes and there is only one study in the literature assessing ideal surface conditioning method with regards to SBS and 3D surface texture changes (12).

This study aimed to determine the ideal method for optimum SBS without causing irreversible surface damage for clinicians by evaluating the effect of surface treatments with the perspectives not only considering shear bond strength of ceramic and metal brackets, but also surface texture changes due to surface conditioning on resin nanoceramic CAD/CAM restorative material. The effect of conditioning with Ti: sapphire Fs laser, Nd: Yag laser, tribochemical coating, and conventional acid etching methods were evaluated using a shear bond test, 3D profilometer, and SEM as surface imaging techniques. The null hypotheses of this study were that type of bracket and type of surface treatment would not affect the SBS of brackets bonded to resin nanoceramics and surface treatment methods would not cause irreversible surface damage to resin nanoceramics.

\section{METHODS}

No ethical approval required for the study because of in vitro design and testing of a dental material already in use. For a power of 0.95 with the significance level $p<0.05$, a sample of at least 15 ceramics for each group would be required based on study by Erdur et al. (5). For this reason, 17 ceramics per group were prepared.

A total number of 170 specimens $(15 \mathrm{~mm} \times 10 \mathrm{~mm} \times 2 \mathrm{~mm}$ ) were prepared from resin nanoceramic CAD/CAM blocks (Cerasmart,
GC Europe, Belgium). These, 170 specimens divided into two groups as metal and porcelain brackets and each group were further divided into five subgroups.

Fs laser group: Selcuk University ILTEK research facility's Ti: sapphire Fs laser ( $\lambda=800 \mathrm{~nm}$; Quantronix, Integra-C-3.5, NY, USA) was used (5). The laser scan was applied five times to the whole geometry. The scan pattern consisted of individual parallel horizontal lines with line gap of $30 \mu \mathrm{m}$. At the end of each line, the laser is automatically switched off and moves to the next line. The fluence for $90 \mathrm{Fs}$ pulse width, $0.05 \mathrm{~W}$ power, and $11 \mathrm{~cm}$ focal length settings was $2,77 \times 10^{1} \mathrm{~J} / \mathrm{cm}^{2}$ (Figure 1a).

A pilot study was conducted to obtain optimum laser parameters for resin nanoceramic specimens to minimize ablation rate and homogenize surface conditioning patterns. Power settings and line gaps changing between 0.05-0.75 W and 10-30-50 $\mu \mathrm{m}$ with five scans were examined by SEM and 3D profilometer. Stated parameters were chosen after the pilot study.

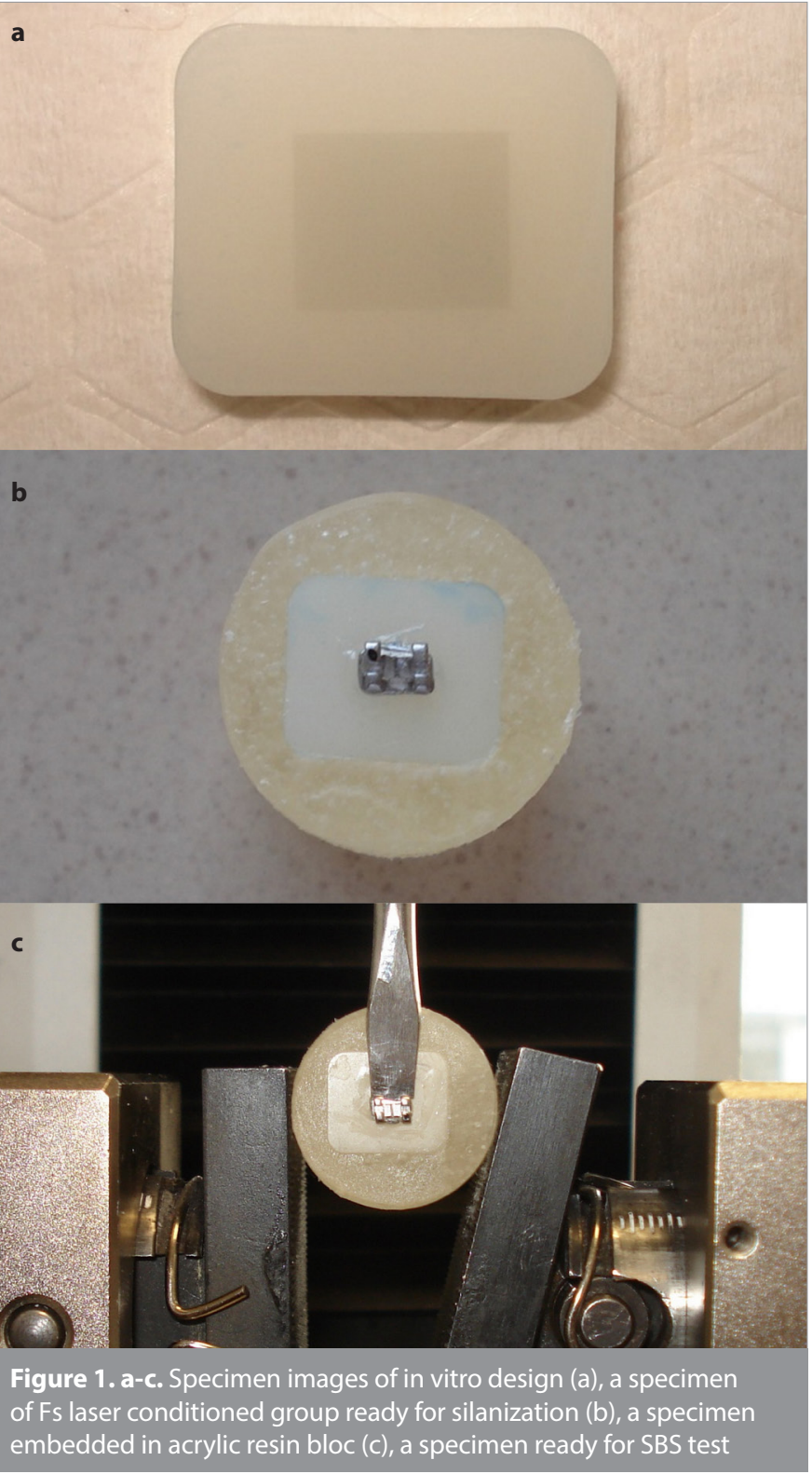


Nd: Yag laser group: Nd: Yag laser (Fotona, Horse Fidelis, Ljubljana, Slovenia) was applied using a 300- $\mu$ m diameter optical fiber perpendicular to the ceramic surface. The output settings were $2 \mathrm{~W}$ and medium-short pulse mode, with 20 seconds application time, at a focal distance of $1 \mathrm{~mm}$ using a noncontact hand piece (5).

Cojet group: Air abrasion with $30-\mu \mathrm{m} \mathrm{Al}_{2} \mathrm{O}_{3}$ particles coated with silica using CoJet System (3M Espe, St. Paul, MN, USA) was applied to the specimens for 5 seconds at a pressure of 2.8 bar (13). The distance between the nozzle and the surface of the ceramic was approximately $10 \mathrm{~mm}$ (14). The sand particle remnants were gently air blown.

HFA group: The ceramic surfaces were etched with $9 \%$ HFA (Porch-Etch, Reliance Orthodontic Products, USA) for 60 seconds. Then, the acid was rinsed off with water for 60 seconds (5).

Orthophosphoric acid (OPA) group: The ceramic surfaces were etched with 37\% OPA (Best Blue Gel, Spot Dis Deposu, Izmir, Turkey) for 60 seconds (6). Then, the acid was rinsed off with water for 60 seconds.

After surface conditioning, silane (Reliance porcelain conditioner, Reliance, IL, U.S.A.) was applied to the treated ceramic surfaces. In total, 85 maxillary central metal brackets (Mini Master Series, American Orthodontics, WI, USA) and 85 porcelain brackets (20/40, American Orthodontics, WI, USA) were bonded randomly with adhesive resin (Transbond XT, 3M Unitek, CA, USA) to the ceramic surfaces. With the aid of a probe, the excess resin was removed. Then, the adhesive resin was light cured for 10 seconds using an LED unit (Valo cordless, Ultradent, USA, standart mode$1000 \mathrm{~mW} / \mathrm{cm}^{2}$ ). All bonding operations were performed by the same operator (M.K.).

After the bonding procedure, the specimens were stored in distilled water at room temparature for at least 24 hours and, then, thermocycled for 1,000 cycles between $5^{\circ} \mathrm{C}$ and $55^{\circ} \mathrm{C}$ with a dwell time 25 seconds in a thermocycler (Julabo, Seelbach, Germany). Then, the specimens were embedded in acrylic resin blocks (Imicryl, Konya, Turkey) (Figure 1b). The SBS test was performed using a universal testing device (Instron 3345, USA) at a crosshead speed of $0.5 \mathrm{~mm} / \mathrm{min}$ (Figure 1c) (11). The force required for debonding was obtained in Newtons $(\mathrm{N})$ and converted into megapascals (MPa). The bracket base areas for the American Orthodontics metal brackets and ceramic brackets were 10.9 and $13.2 \mathrm{~mm}^{2}$, respectively.

After surface roughening, the average surface roughness of chosen conditioned and nonconditioned ceramic specimens were evaluated with a profilometer (AEP NanoMap-LS, CA, USA). A diamond stylus (tip radius, $5 \mu \mathrm{m}$ ) acted across the surface under a continuous charge of $40 \mathrm{mg}$ with a speed of $40 \mu \mathrm{m} / \mathrm{s}$ and a range of $500 \mu \mathrm{m}$, to measure the roughness value in $\mu \mathrm{m}$. For each measurement, the device performs random 30 probing around the perimeter of a $500-\mu \mathrm{m}$ line and calculates mean roughness. Two extra specimens from each surface conditioning group with two measurements were planned at the beginning of the study. However, after obtaining elevated SBS results, the addition of a nonconditioned specimen with four measurement was decided for 3D profilometer evaluation to clarify the exact surface texture changes. In total, means of four measurements from each group were calculated from the center area of specimens. For Fs laser group, two additional measurements for each specimen were made from the laser conditioned border area to determine the ablation amount. No sample size calculation has been conducted for profilometric evaluation.

Following surface roughening, one specimen from each conditioned and nonconditoned groups was selected randomly, and all specimens were sputter-coated with gold-palladium and evaluated using a SEM (Jeol JMS-6390LV, Jeol, Tokyo).

After SBS test, the ceramic surfaces underwent surface morphological analyses using a stereomicroscope (Leica M50, Wetzlar/ Germany) at $25 \times$ magnification to determine the amount of composite resin remaining according to the Adhesive Remnant Index (ARI) proposed by Artun and Bergland (15) scoring as: 0-no adhesive remaining on the ceramic surface; 1 -less than $50 \%$ adhesive remaining on the ceramic; 2 -more than $50 \%$ adhesive remaining on the ceramic; and $3-100 \%$ adhesive remaining on the ceramic, with a distinct impression of the bracket mesh.

Any damage that may have occured to the ceramic surfaces during SBS testing was recorded using the Porcelain Fracture Index (PFI) proposed by Bourke and Rock (16) scoring as: 0-ceramic surface intact or in the same condition as before the bonding; 1-surface damage limited to very superficial ceramic; 2-surface damage which features significant loss of ceramic requiring restoration of the defect by composite resin or replacement of the restoration; and 3-surface damage where the core material has been exposed.

For the Statistical Package for Social Sciences, version 15.0 software (SPSS Inc.; Chicago, IL, USA). Kolmogorov-Smirnov Test was used to verify normality distrubution of test values. Two-way analysis of variance (ANOVA) was used to check the effect of type of bracket, type of surface treatment, and their interaction. Tukey's honestly significantly different test was used to compare SBS among the groups. Chi-square $\left(X^{2}\right)$ test was used to determine if there were any significant differences in the ordinal ARI and PFI values. Nonparametric Wilcoxon signed-rank test was used to compare surface roughness values. Statistical significance was set at the 0.05 probability level.

\section{RESULTS}

According to two-way ANOVA type of bracket, type of surface treatment and their interaction significantly affected the SBS test (Table 1).

The SBS for the study groups are shown in Table 2. The mean SBS values of the metal bracket groups ranged from 8.66 to 13.02 MPa. HFA, Cojet, and Fs laser groups have significantly higher mean SBS than Nd: Yag laser group. The mean SBS values of the porcelain bracket groups ranged from 9.36 to $10.91 \mathrm{MPa}$. There were no significant differences between study groups. Except for Nd: Yag laser group, all metal bracket groups showed 
Table 1. Two-way ANOVA results for the type of bracket, type of treatment, and the interaction terms according to bond strength data (MPa).

\begin{tabular}{|lccccc|}
\hline Effect & df & Sum of squares & Mean square & F ratio & p \\
\hline Bracket type & 1 & 97.799 & 97.799 & $.001^{*}$ & .095 \\
Surface conditioning & 4 & 150.517 & 37.629 & 4.654 & $.001^{*}$ \\
Bracket type x surface conditioning & 4 & 138.795 & 34.699 & 4.291 & $.003^{*}$ \\
\hline${ }^{*} p<0.05$ & & & & & \\
\hline
\end{tabular}

Table 2. Mean, standard deviation (SD), minimum (min.)-maximum (max.), and median of the SBS (MPa) according to the surface treatments.

\begin{tabular}{|c|c|c|c|c|c|c|c|c|}
\hline Surface treatment & Metal bracket & SD & Min.-Max. & Median & Porcelain bracket & SD & Min.Max. & Median \\
\hline Orthophosphoric acid & $10.32 A^{B C}$ & 3.65 & $15.86-2.96$ & 10.37 & $9.36^{\mathrm{Ac}}$ & 2.81 & $4.91-12.64$ & 9.55 \\
\hline Hydrofluoric acid & $13.02^{\mathrm{Ac}}$ & 2.27 & $9.38-15.96$ & 13.20 & $10.91^{\mathrm{Ac}}$ & 1.60 & $13.34-8.66$ & 10.84 \\
\hline Nd:Yag laser & $8.66^{\mathrm{BC}}$ & 2.13 & $12.57-4.41$ & 8.56 & $10.38^{\mathrm{AC}}$ & 1.98 & $6.81-13.20$ & 10.44 \\
\hline Fs laser & $13.01^{\mathrm{Ac}}$ & 2.43 & $9.31-16.63$ & 12.72 & $9.68^{\mathrm{Ad}}$ & 1.95 & $5.64-12.44$ & 9.65 \\
\hline
\end{tabular}

Table 3. Frequency distribution of adhesive remnant index (ARI)

scores.

\begin{tabular}{llccc|} 
& \multicolumn{5}{c|}{ ARI scores } \\
\cline { 2 - 5 } Group & $\mathbf{0}$ & $\mathbf{1}$ & $\mathbf{2}$ & $\mathbf{3}$ \\
\hline Metal bracket & & & & \\
Orthophosphoric acid & 0 & 7 & 2 & 8 \\
Hydrofluoric acid & 0 & 13 & 1 & 3 \\
Cojet & 1 & 9 & 0 & 7 \\
Nd:Yag laser & 15 & 1 & 0 & 1 \\
Fs laser & 3 & 4 & 3 & 7 \\
Porcelain bracket & & & & \\
Orthophosphoric acid & 1 & 12 & 2 & 2 \\
Hydrofluoric acid & 0 & 8 & 9 & 0 \\
Cojet & 0 & 7 & 7 & 3 \\
Nd:Yag laser & 7 & 8 & 2 & 0 \\
Fs laser & 0 & 7 & 6 & 4 \\
\hline 0-no adhesive remaining on the ceramic surface \\
1-less than 50\% adhesive remaining on the ceramic surface \\
2-more than 50\% adhesive remaining on the ceramic surface \\
3-100\% adhesive remaining on the ceramic surface, with a distinct impression \\
of the bracket mesh
\end{tabular}

Table 4. Frequency distribution of porcelain fracture index (PFI) scores.

\begin{tabular}{|c|c|c|c|c|}
\hline \multirow[b]{2}{*}{ Group } & \multicolumn{4}{|c|}{ PFI scores } \\
\hline & 0 & 1 & 2 & 3 \\
\hline \multicolumn{5}{|l|}{ Metal bracket } \\
\hline Orthophosphoric acid & 13 & 1 & 3 & 0 \\
\hline Hydrofluoric acid & 16 & 1 & 0 & 0 \\
\hline Cojet & 11 & 2 & 4 & 0 \\
\hline Nd:Yag laser & 17 & 0 & 0 & 0 \\
\hline Fs laser & 8 & 2 & 7 & 0 \\
\hline \multicolumn{5}{|l|}{ Porcelain bracket } \\
\hline Orthophosphoric acid & 13 & 2 & 2 & 0 \\
\hline Hydrofluoric acid & 15 & 1 & 1 & 0 \\
\hline Cojet & 8 & 3 & 6 & 0 \\
\hline Nd:Yag laser & 16 & 1 & 0 & 0 \\
\hline Fs laser & 4 & 5 & 8 & 0 \\
\hline \multicolumn{5}{|c|}{$\begin{array}{l}\text { 0-ceramic surface intact or in the same condition as before the bonding } \\
\text { 1-surface damage limited to very superficial ceramic } \\
\text { 2-surface damage which features significant loss of ceramic requiring resto- } \\
\text { ration of the defect by composite resin or replacement of the restoration } \\
\text { 3-surface damage where the core material has been exposed }\end{array}$} \\
\hline
\end{tabular}

Table 5. Mean, standard deviation (SD), minimum (min.)-maximum ( $\max$ ), and median values of surface roughness ( $\mu \mathrm{m}$ ) according to the surface treatments

\begin{tabular}{|lcccc|}
\hline Group & Mean surface roughness & SD & Min.-Max. & Median \\
\hline No treatment & $0.031^{\mathrm{a}}$ & 0.006 & $0.021-0.036$ & 0.033 \\
Orthophosphoric acid & $0.084^{\mathrm{b}}$ & 0.009 & $0.071-0.092$ & 0.088 \\
Hydrofluoric acid & $0.108^{\mathrm{b}}$ & 0.024 & $0.084-0.140$ & 0.104 \\
Cojet & $0.690^{\mathrm{c}}$ & 0.143 & $0.515-0.823$ & 0.711 \\
Nd:Yag laser & $0.100^{\mathrm{b}}$ & 0.009 & $0.092-0.112$ & 0.097 \\
Fs laser & $0.309^{\mathrm{d}}$ & 0.029 & $0.286-0.347$ & 0.301 \\
\hline \multicolumn{4}{l}{ Mean values represented with different superscript lower-case letter are significantly different according to Wilcoxon signed-rank test (p<0.05) } \\
\hline
\end{tabular}

higher mean SBS compared with porcelain bracket groups. This difference reached statistical significance only in Fs laser group $(p<0.05)$.
Bond failure modes are shown in Table 3. Except for Nd: Yag porcelain/OPA porcelain groups $(p>0.05)$, the ARI scores showed that adhesive failures between the ceramic and com- 


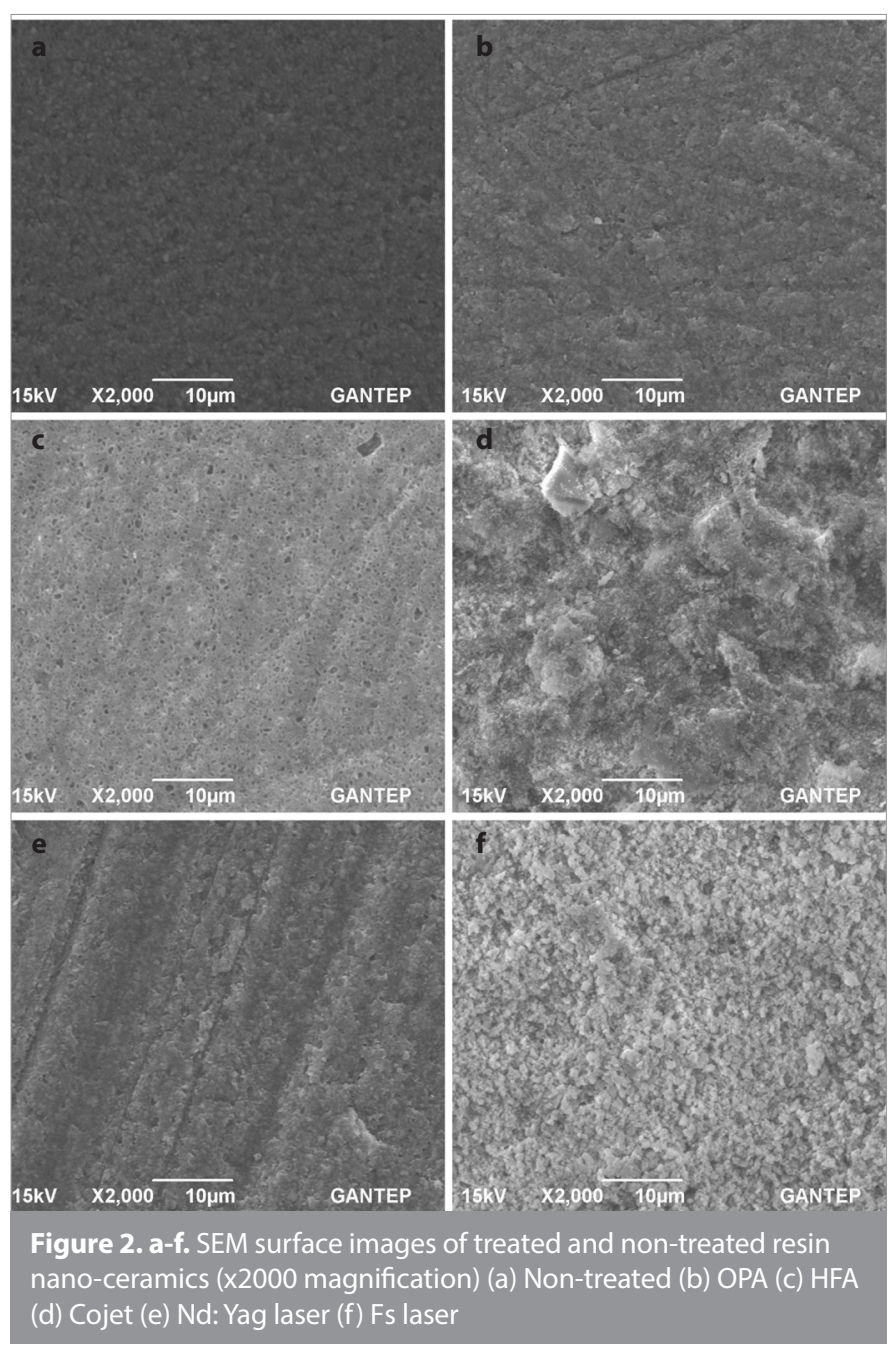

posite resin were the predominant mode of failure in $\mathrm{Nd}$ : Yag laser groups $(p<0.05)$. For other study groups, a failure mode shift between composite resin and bracket base were observed especially in Fs laser groups compared with $\mathrm{Nd}$ : Yag laser groups $(p<0.05)$.

Porcelain fracture modes are shown in Table 4. Except for Cojet metal/Nd: Yag porcelain groups ( $p>0.05$ ), the highest incidence of cohesive fracture of resin nanoceramics were observed in Fs laser and Cojet groups, the lowest incidence observed in Nd: Yag laser groups $(p<0.05)$.

Representative SEM images of the treated and nontreated resin nanoceramics are presented in Figure 2. Homogenous microporous surface texture of the nontreated specimen showed a variation in the surface microstructure with the surface treatments. Due to the partial dissolving of organic component of resin nanoceramic, OPA showed minimal erosive areas. HFA effecting both organic and inorganic components of resin nanoceramic showed erosive areas and randomly distributed micropores. Silica-coating produced random surface peeling and well-defined microsize elevation and depression areas. Nd: Yag laser produced undulated surface morphology with sulcular appearance. Fs laser produced uniform micro and nano roughnesses without crack formation on the surface.
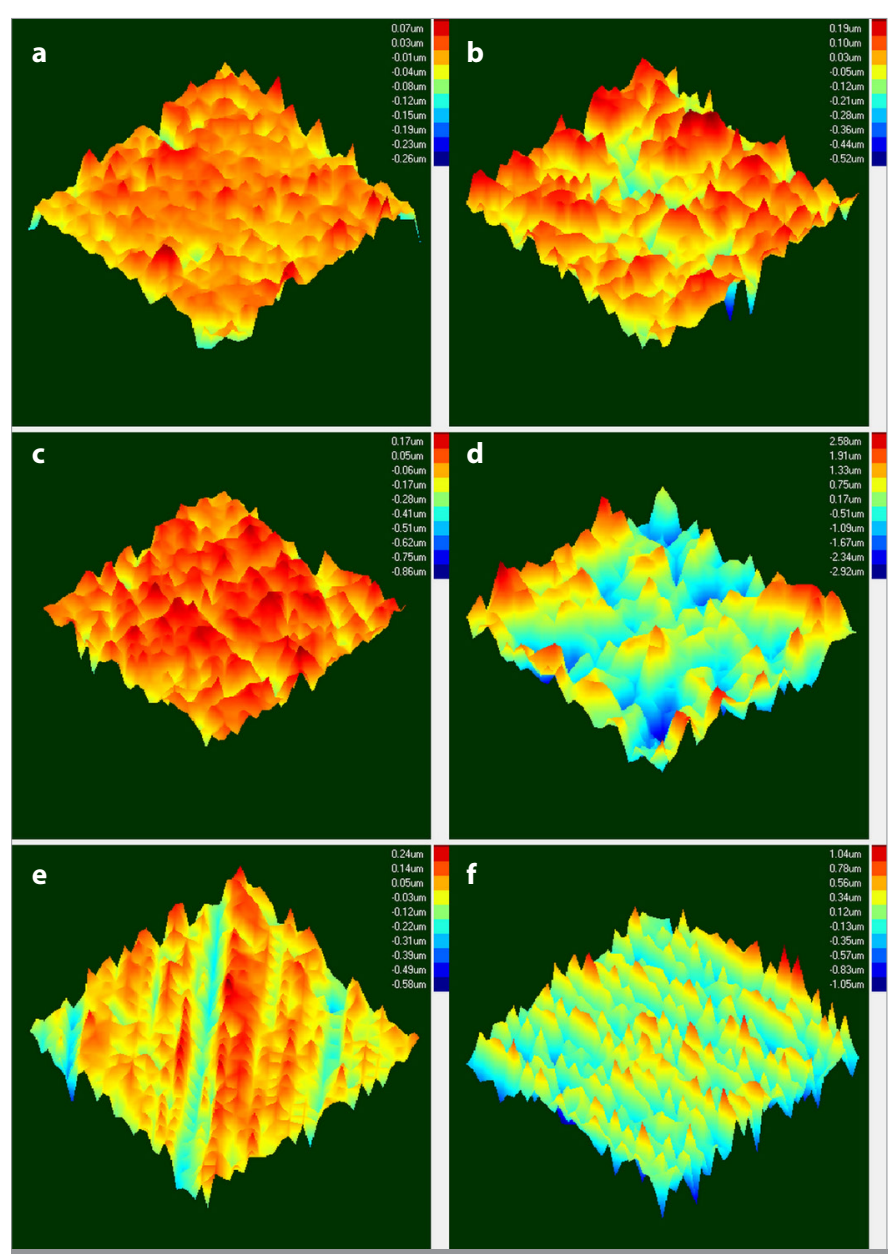

Figure 3. a-f. 3D profilometer images of treated and nontreated resin nanoceramics, (a) Nontreated (Maximum height $0.092 \mu \mathrm{m}$, minimum height $-0.264 \mu \mathrm{m}$ ). (b) OPA (Maximum height $0.265 \mu \mathrm{m}$, minimum height $-0.517 \mu \mathrm{m}$ ). (c) HFA (Maximum height $0.281 \mu \mathrm{m}$, minimum height $-0.483 \mu \mathrm{m}$ ). (d) Cojet (Maximum height $2.472 \mu \mathrm{m}$ minimum height $-2.924 \mu \mathrm{m}$ ). (e) Nd: Yag laser (Maximum height $0.325 \mu \mathrm{m}$, minimum height $-0.461 \mu \mathrm{m}$ ). (f) Fs laser (Maximum height $0.959 \mu \mathrm{m}$, minimum height $-1.051 \mu \mathrm{m})$.
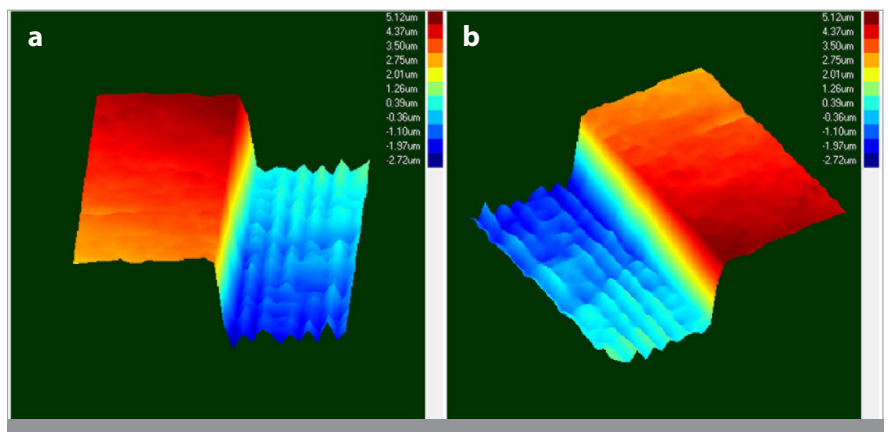

Figure 4. a-b. Images of ablated and nonablated border area of Fs laser trated resin nanoceramic from different angles. Maximum height $5.116 \mu \mathrm{m}$, minimum height $-2.348 \mu \mathrm{m}$. Peak to peak difference $7.463 \mu \mathrm{m}$.

Representative 3D profilometer images of the conditioned and nonconditioned resin nanoceramics are presented in Figure 3. 3D profilometer images of laser conditioned and nonconditioned border area of Fs laser treated resin nanoceramic are presented in Figure 4. Compared to nonconditioned specimen minimum and maximum height changes can be clearly seen in 
Figure 3 caused by surface conditioning methods. Cojet produced the highest increase of minimum and maximum height levels, which can be speculated as a sign of maximum increase of surface roughness (Figure 3d). OPA, HFA, and Nd: Yag laser groups showed comparable minimum and maximum height changes, which could be interpreted as a sign of similar surface roughness values (Figures $3 b, 3 c$, and $3 e$ ). Specific to Fs laser group, $\sim 5 \mu \mathrm{m}$ ablation was observed at the border of the laser conditioned and nonconditioned areas (Figure 4).

Mean roughness values $(\mu \mathrm{m})$ are shown in Table 5 . All surface conditioning methods caused significant increase in surface roughness compared with nonconditioned specimen according to Wilcoxon signed-rank test. The surface roughnesses of the conditioned resin nanoceramics indicated significant differences among the groups $(p<0.05)$. Cojet caused a significantly higher mean surface roughness compared with other surface treatments. Fs laser midsection roughness value is significantly lower than Cojet and significantly higher than OPA, HFA, and Nd: Yag laser groups $(p<0.05)$. There was no statistically significant difference between OPA, HFA, and Nd: Yag laser groups ( $p>0.05$ ).

\section{DISCUSSION}

The results of this study demonstrated no significant SBS differences between the metal and ceramic brackets; therefore, the first null hypothesis was accepted. The results of this study demonstrated significant SBS differences among surface conditioning groups; therefore, the second null hypothesis was rejected. The results of this study demonstrated significant surface texture changes depending on conditioning methods; therefore, the third null hypothesis was rejected.

CAD/CAM systems registered a constantly increasing use in dentistry. This technology allows a completely digital workflow, from impression to final framework, with acceptable clinical reliability (17), good aesthetics (18), and excellent patients feedback (19). However, only few studies tested the bond strength of orthodontic appliances on these recently introduced materials (13); hence, this study was planned and performed to specify the most reliable surface treatment method for bonding ceramic and metal brackets onto resin nanoceramic CAD/CAM material with regards to SBS values for bonded brackets, ARI and PFI scores, SEM images, and 3D profilometer analyses. Thermocycling procedure was applied to the specimens before SBS testing as a screening procedure for the performance of the bonded interfaces under standardized hydrothermal stresses (13). SBS test was used to evaluate the bond strength between resin nanoceramic material and brackets. Although the clinical implications of in vitro studies are questionable, they are the first choice in testing new materials and methods before they can be used in vivo.

In accordance with previous studies, before bonding procedure, a silane coupling agent was applied to increase wettability and the bonding of resin to ceramics after surface conditioning (1).

In the literature, there are no clear guidelines about shear force limits, but in fact a good orthodontic biomaterial should allow good adhesion in order to sustain masticatory forces (5-10 MPa). In contrast, adhesion forces should not be too strong in order to avoid enamel loss after debonding (40-50 MPa). Therefore, the ideal orthodontic biomaterial should have bonding forces included in the interval of 5-50 MPa for enamel bonding, even if these limits are mostly theoretical (20). But for ceramic restorations, it was reported that bond strength values over $13 \mathrm{MPa}$ between the ceramic and the composite resin increase the risk of cohesive fractures (21). For this reason, achieving the highest SBS may not be the most significant clinical factor. The clinicians should prefer ideal bonding methods that can endure masticatory and orthodontic forces during treatment and easily debonded at the end of the treatment. In this study, all surface treatment groups acquired optimum or higher mean SBS. Porcelain bracket groups showed lower mean SBS than metal bracket groups other than Nd: Yag laser group, this difference reached statistical significance only in Fs laser group ( $p<0.05 ; 9.68 \pm 1.95 \mathrm{MPa}$ and $13.01 \pm 2.43 \mathrm{MPa}$, respectively). It was shown that ceramic brackets promote higher SBS than metal brackets (13). Differences in bonding materials/adhesives, different settings of load application, and different material preparation techniques might be the reason for variation of the results in this study.

In the literature, different ceramic materials have been subjected to surface conditioning with different laser types, and acceptable results have been reported regarding SBS of orthodontic brackets $(5,11,12)$. There are no optimized laser processing parameters for each material to be tested because of unique microstructure configurations and chemical compositions of the materials (22). For this reason, we conducted a pilot study for optimum Fs laser parameters both to attain minimal ablation rate and uniform surface morphology for bonding. Laser settings of five scans, a laser pulse power of $0.05 \mathrm{~W}$, and line gap of $30 \mu \mathrm{m}$ were chosen. Using these optimized parameters, $\sim 5 \mu \mathrm{m}$ ablation was observed in 3D profilometer evaluation (Figure 4). We did not observe melted and scattered particles and cracks because of the Fs laser conditioning of resin nanoceramics in SEM and 3D profilometer analyses. This observation showed the notable lack of heating and shockwave effects of Fs laser on resin nanoceramic. Absence of microcracks with uniform micro and nano roughnesses on resin nanoceramic surface after Fs laser irradiation might contribute to higher SBS (Figures $2 f$ and $3 f$ ).

Erdur et al. (5) performed in vitro study on feldspathic and e-Max surfaces using Ti: sapphire Fs, Nd: Yag, and Er: Yag lasers, and reported that the highest SBS was obtained with Ti: sapphire Fs laser conditioning. Akpinar et al. (23) also reported higher SBS for Ti: sapphire Fs laser on feldspathic porcelain surfaces. Aglarci et al. (24) compared SBS of extracted human premolar teeth conditioned with Ti: sapphire Fs laser and Er: Yag laser using metal brackets and reported lower SBS for Ti:sapphire Fs laser group, probably from single laser scan regime. Although using different power settings owing to material differences, our results are in line with Erdur et al. (5) and Akpinar et. al. (23)

Although some of the researchers have reported that Nd: Yag laser conditioning is an effective technique for bracket adhesion to porcelain surfaces $(25,26)$, there are other researchers who stated insufficent bond strengths with $\mathrm{Nd}$ : Yag laser $(5,12)$. In this study, Nd: Yag laser conditioning caused optimum SBS and lower PFI scores. Hybrid compound of organic and inorganic particles 
in resin nanoceramics might render it susceptible to laser penetration and responsible for causing optimum SBS values.

Cojet System uses 30- $\mu \mathrm{m} \mathrm{Al}_{2} \mathrm{O}_{3}$ particles coated with silica for surface modification. With the aid of high-pressure deposition of these particles on the substrate surface are provided (13). A covalent bonding between silica-coated substrate and composite resin was established with the usage of Cojet System (2). In this study, silica coating followed by silane application fulfilled the required threshold. The results are in aggreement with the previous studies $(2,4,13)$, but high cost is the main disadvantage of tribochemical coating. Moreover, extra precautions such as rubber dam usage and high-power suction systems are recommended to avoid soft tissue injuries and spread of particles, respectively (27).

It was found that HF acid etching was an acceptable method for ceramic surface conditioning (2). However, $\mathrm{HF}$ acid is responsible for dissolving the glass component of the silica-based ceramics, and there is no effect of $\mathrm{HF}$ acid on the high-strength materials such as zirconia and core ceramics $(11,28)$. In this study, HFA etching dissolved both organic and inorganic compounds of the resin nanoceramic material and created a uniform microporous surface morphology amenable to penetration of silan and adhesive resin (Figure $2 \mathrm{c}$ ). Its surface roughness values were comparable to OPA group and lesser than Cojet and Fs laser groups (Figure 3 and Table 5). HFA proved to be an effective surface conditioning method for resin nanoceramics, but it is a highly toxic and corrosive material, so maximum precautions should be taken such as protective clothing and safety goggles to avoid skin and eye contact (29). We found that OPA-etched groups had similar SBS to those that are HFA-etched. Surface modification effect of OPA can be clearly seen on SEM images and 3D profilometer analysis (Figures $2 \mathrm{~b}$ and $3 \mathrm{~b}$ ). The possible reason for acceptable SBS of OPA etched groups was the increased micromechanic retention because of the modification of polymer containing organic component of the resin nanoceramic. Backer et al has shown that resin composite CAD/CAM materials are susceptible to roughening in acid conditions, which differs from conventional ceramics (30). Further studies are needed to clarify the exact role of OPA on resin nanoceramics. Main advantages of both OPA and HFA conditioning to laser systems or air borne particle abrasion are their cost effectiveness and ease of application.

In this study, ARI scores did not show correlation with the bond strength. The groups with similiar SBS had different ARI scores, so one cannot define the site of bond failure with a definite threshold of shear force (2). But, mean SBS increases could imply a failure mode shift from ceramic and composite resin to composite resin and bracket interface.

All surface conditioning methods investigated in this study have sufficent SBS to withstand forces during orthodontic treatment. In fact, higher SBS exibited by Cojet and Fs laser groups showed higher PFI scores. Lawson et al (31) suggested polymer containing CAD/CAM materials might not possess high initial strength. Goujat et al (7) stated lower fracture toughness values for resin nanoceramics. These facts can elucidate high PFI scores seen in our study that resin nanoceramic could not withstand concentric high pressure during debonding. Due to high PFI scores obtained in this study, extra precautions should be taken for debonding of resin nanoceramics.

Regarding the limited amount of studies evaluating surface roughness of conditioned porcelain surfaces, Erdur et al (32) reported Fs laser treated group having the highest mean roughness value. Interestingly, using a 5\% HFA just for 20 seconds on IPS e-Max surfaces, they also reported $2.21 \mu \mathrm{m}$ mean surface roughness, which was much higher than HFA group of this study. Other than different measurement techniques and different glass components of study materials, they also did not include a "no treatment" group, making impossible to know roughness value of the test material before surface conditioning, therefore, any comparisons between the studies might be arbitrary. Çevik et al. (12) reported no significant differences among control, OPA, or HFA treated surfaces of lithium disilicate and feldspathic ceramics. In this study, mean surface roughness values of OPA and HFA were similiar and significantly higher than the control group. Conditioning protocol, material, and measurement differences could be speculated as possible causative factors. In this study, Cojet produced the highest mean surface roughness while Fs laser came behind. These values are the means of midsection measurements none of the surface conditioning groups caused ablation other than Fs laser group. If $\sim 5 \mathrm{~m}$ ablation detected on border area of Fs laser group added to the calculation the highest mean surface roughness ought to be the Fs laser group (Figure 4). Surface roughness values of Nd: Yag, HFA, and OPA groups were in acceptable range. Although on $\mu \mathrm{m}$ level, roughness values of Fs laser and Cojet groups might rise concerns of patients following debonding especially for minimally prepared anterior full crown restorations and might require repairs or renewals.

\section{CONCLUSION}

Within the limitations of this in vitro study, the following conclusions can be made.

- HFA is an effective method for surface conditioning of resin nanoceramic material as stated before for other ceramic materials. OPA combined with silane application caused optimum SBS and can be used as an alternative to HFA.

- Surface conditioning with Nd: Yag laser provided optimum SBS and PFI scores for resin nanoceramic material.

- Although surface conditioning of resin nanoceramic material with Fs laser and Cojet treatment provided optimum or higher SBS, increase in ceramic fracture risk during debonding, possible aesthetic failures because of elevated micro roughness and high cost are important disadvantages of these methods. Moreover, requirement of a pilot study for optimum Fs laser dosage is another disadvantage of Fs laser usage. Clinicians planning to use Cojet or Fs laser for surface conditioning of hybrid ceramics should approach with caution and limit their usage only for specific cases, which demand higher SBS and previously informing the patient about risk of failure.

Peer-review: Externally peer-reviewed. 
Author Contributions: Conception - M.K., M.D.; Design - M.K., Ö.D.; Supervision - M.D.; Data Collection and/or Processing - M.K., Ö.D.; Analysus and/or Interpretation - M.K., M.D.; Writing Manuscript - M.K.; Critical Review - M.D., Ö.D.; Literature Search - M.K., Ö.D.

Conflict of Interest: The author has no conflict of interest to declare.

Financial Disclosure: This Project Supported by Dicle University Scientific Research Projects (Project Number: Dis 17-010).

Acknowledgement: The authors thank to Prof.Dr Hamdi Şükür Kılıç and his assistant Yasemin Gündoğdu for their valuable information and supports on Fs laser.

\section{REFERENCES}

1. Blatz MB, Sadan A, Kern M. Resin-ceramic bonding: a review of the literature. J Prosthet Dent 2003; 89: 268-74. [Crossref]

2. Karan S, Büyükyilmaz T, Toroğlu MS. Orthodontic bonding to several ceramic surfaces: are there acceptable alternatives to conventional methods? Am J Orthod Dentofac Orthop 2007; 132: 7-14. [Crossref]

3. Turk T, Sarac D, Sarac YS, Elekdag-Turk S. Effects of surface conditioning on bond strength of metal brackets to all-ceramic surfaces. Eur J Orthod 2006; 28: 450-6. [Crossref]

4. Schmage P, Nergiz I, Herrmann W, Özcan M. Influence of various surface-conditioning methods on the bond strength of metal brackets to ceramic surfaces. Am J Orthod Dentofac Orthop 2003; 123: 5406. [Crossref]

5. Erdur EA, Basciftci FA. Effect of Ti:sapphire laser on shear bond strength of orthodontic brackets to ceramic surfaces. Lasers Surg Med 2015; 47: 512-9. [Crossref]

6. An KM Sohn DS. The effect of using laser for ceramic bracket bonding of porcelain surfaces. Korean J Orthod 2008; 38: 275-82. [Crossref]

7. Goujat A, Abouelleil H, Colon P, Jeannin C, Pradelle N, Seux D, et al. Mechanical properties and internal fit of 4 CAD-CAM block materials. J Prosthet Dent. 2018; $119: 384-9$. [Crossref]

8. Coldea A, Swain MV, Thiel N. Mechanical properties of polymer-infiltrated-ceramic-network materials. Dent Mater 2013; 29: 419-26. [Crossref]

9. Ruse ND, Sadoun MJ. Resin-composite blocks for dental CAD/CAM applications. J Dent Res 2014; 93: 1232-4. [Crossref]

10. Nguyen JF, Migonney V, Ruse ND SM. Resin composite blocks via high-pressure high-temperature polymerization. Dent Mater 2012; 28: 529-34. [Crossref]

11. Cevik P, Eraslan O, Eser K, Tekeli S. Shear bond strength of ceramic brackets bonded to surface-treated feldspathic porcelain after thermocycling. Int J Artif Organs 2018; 41: 160-7. [Crossref]

12. Cevik P, Karacam N, Eraslan O, Sari Z. Effects of different surface treatments on shear bond strength between ceramic systems and metal brackets. J Adhes Sci Technol 2017; 31: 1105-15. [Crossref]

13. Elsaka SE. Influence of surface treatments on bond strength of metal and ceramic brackets to a novel CAD/CAM hybrid ceramic material. Odontology 2016; 104: 68-76. [Crossref]

14. Ozcan M. The use of chairside silica coating for different dental applications: a clinical report. J Prosthet Dent 2002; 87: 469-72. [Crossref]
15. Artun J, Bergland S. Clinical trials with crystal growth conditioning as an alternative to acid-etch enamel pretreatment. Am J Orthod Dentofac Orthop 1984; 85: 333-40. [Crossref]

16. Bourke BM, Rock WP. Factors Affecting the Shear Bond Strength of Orthodontic Brackets to Porcelain. Br J Orthod 1999; 26: 285-90. [Crossref]

17. Alessandretti $R$, Borba $M$, Benetti $P$, Corazza PH, Ribeiro R, Della Bona A. Reliability and mode of failure of bonded monolithic and multilayer ceramics. Dent Mater. 2017; 33: 191-7. [Crossref]

18. Della Bona A, Pecho OE, Ghinea R, Cardona JC, Pérez MM. Colour parameters and shade correspondence of CAD-CAM ceramic systems. J Dent 2015; 43: 726-34. [Crossref]

19. Sfondrini MF, Gandini P, Malfatto M, Di Corato F, Trovati F, Scribante A. Computerized casts for orthodontic purpose using powder-free intraoral scanners: accuracy, execution time, and patient feedback. Biomed Res Int 2018; 4103232. [Crossref]

20. Scribante A, Contreras-Bulnes R, Montasser MA, Vallittu PK. Orthodontics: Bracket Materials, Adhesives Systems, and Their Bond Strength. Biomed Res Int. 2016; 1329814. [Crossref]

21. Thurmond JW, Barkmeier WW, Wilwerding TM. Effect of porcelain surface treatments on bond strengths of composite resin bonded to porcelain. J Prosthet Dent 1994; 72: 355-9. [Crossref]

22. Ji L, Li L, Devlin H, Liu Z, Jiao J, Whitehead D. Ti: Sapphire femtosecond laser ablation of dental enamel, dentine, and cementum. Lasers Med Sci 2012; 27: 197-204. [Crossref]

23. Akpinar YZ, Irgin C, Yavuz T, Aslan MA, Kilic HS, Usumez A. Effect of femtosecond laser treatment on the shear bond strength of a metal bracket to prepared porcelain surface. Photomed Laser Surg 2015; 33: 206-12. [Crossref]

24. Aglarci C, Demir N, Aksakalli S, Dilber E, Sozer OA, Kilic HS. Bond strengths of brackets bonded to enamel surfaces conditioned with femtosecond and Er:YAG laser systems. Lasers Med Sci 2016; 31: 1177-83. [Crossref]

25. Hosseini MH, Sobouti F, Etemadi A, Chiniforush N, Shariati M. Shear bond strength of metal brackets to feldspathic porcelain treated by Nd:YAG laser and hydrofluoric acid. Lasers Med Sci 2015; 30: 837-41. [Crossref]

26. Poosti M, Jahanbin A, Mahdavi P, Mehrnoush S. Porcelain conditioning with Nd:YAG and Er:YAG laser for bracket bonding in orthodontics. Lasers Med Sci 2012; 27: 321-4. [Crossref]

27. Galia Reston E, Filho Calabrez S, Arossi G, Barreto Cogo R, Dos Santos Rocha C, Quadrado Closs L. Repairing ceramic restorations: final solution or alternative procedure? Oper Dent 2008; 33: 461-6. [Crossref]

28. Zhang ZC, Qian YF, Yang YM, Feng QP, Shen G. Bond strength of metal brackets bonded to a silica-based ceramic with light-cured adhesive: influence of various surface treatment methods. J Orofac Orthop 2016; 77: 366-72. [Crossref]

29. Ozcan M, Allahbeickaraghi A, Dundar M. Possible hazardous effects of hydrofluoric acid and recommendations for treatment approach: a review. Clin Oral Investig 2012; 16: 15-23. [Crossref]

30. Backer AD, Eliseu AM, Eckert GJ. Effects of simulated gastric juice on CAD/CAM resin composites-morphological and mechanical evaluations. J Prosthodont 2017; 26: 424-31. [Crossref]

31. Lawson NC, Bansal R, Burgess JO. Wear, strength, modulus and hardness of CAD/CAM restorative materials. Dent Mater 2016; 32: 275-83. [Crossref]

32. Erdur EA, Basciftci FA. Effect of ti: sapphire-femtosecond laser on the surface roughness of ceramics. Lasers Surg Med 2015; 47: 833-8. [Crossref] 